\title{
Modular Approach to 3D Representation of Underground Infrastructure in the Model for Underground Data Definition and Integration (MUDDI)
}

\author{
J. Lieberman ${ }^{1}$, C. Roensdorf ${ }^{2}$ \\ ${ }^{1}$ Open Geospatial Consortium - jlieberman@ogc.org \\ ${ }^{2}$ Ordnance Survey Limited, Adanac Drive, Nursling, Southampton, SO16 0AS - Carsten.Roensdorf@os.uk
}

KEY WORDS: data model, 3D, infrastructure, OGC, standards, underground, utility, built environment

\begin{abstract}
:
Data and models of the built environment enable urban systems to serve their inhabitants and adapt to ever increasing rates of change in society, climate, and. Models of the built environment at or above the ground surface need to be 3 dimensional to best fill that role, but the subsurface also has critical 3-dimensional properties that are even more difficult to characterize being hidden from view. There are many compelling use cases for high quality data on the underground environment at varying levels of detail, from which a list of 6 critical use cases are presented here. Data on the location and disposition of buried utility are difficult to collect and maintain, but the value of avoiding damage, delay, injury and cost with good underground data in all these cases far outstrips the cost and difficult of obtaining it. Effective management and utilization of underground data also depend on models and schemas to organize them. Sharing and exchange of such data require standard models that are agreed between data providers and consumers. There are presently a number of applicable models and standards, but they often reflect a specific perspective, focus, and priorities that make it difficult for any one of them to provide a holistic awareness of the entire underground built environment at the multiple levels of complexity required by the use cases. The draft Model for Underground Data Definition and Integration (MUDDI), a comprehensive integration model for underground information takes a modular approach, with a conceptual core that covers basic geometric representations of underground assets, and a number of extension modules that add more specialized capabilities as well as interfaces with existing models. Several prototyping efforts have generated physical implementations of the MUDDI conceptual model and application deployments populated by operational utilities data, in particular the NUAR and LUAR pilot projects sponsored by the UK Geospatial Commission. An Open Geospatial Consortium Standards Working Group (SWG) is being formed to build on the draft MUDDI model as well as the experiences gained in pilot projects, in order to publish a full specification of the model at the conceptual, logical, and physical levels. Another SWG objective will be to create a roadmap of critical extension modules, particularly those which support upcoming 3D-4D digital twin technologies for visualization, operation, and simulation. Other advanced use cases for these extensions, such as mixed reality visualization and navigation, are expected to become common as both the demands on our built environment and the data available to manage it continue to expand.
\end{abstract}

\section{INTRODUCTION}

Every year thousands of otherwise routine street excavations around the world go astray for lack of usable information about the underground utility infrastructure they encounter. Largescale construction projects end up stalled, incurring delay claims and change orders that significantly increase costs and risk, because the locations of utility installations were never properly measured, recorded or represented. Bid costs may well be $10-30 \%$ higher than need to allow for contingencies to deal with this lack of knowledge. Poor data about underground built environment dependencies and vulnerabilities often stands in the way of effective disaster response and recovery. These costs and risks would be unnecessary if accurate, comprehensive information on the underground built environment were available and shared between responsible parties for rapid integration and analysis. The first step towards achieving this capability involves developing geo-enabled utility data models that enable data interoperability and integration. Development and adoption of such models would deliver significant benefits by improving data interchange, integration, and application readiness. The models should focus on those attributes most important for specific use cases such as safe digging, construction design, and disaster resilience, incorporating model extensions as needed to accommodate more complex use cases as the business value of data sharing and interoperability is more fully established.

An additional value of developing standardized data models for selected underground utility components and environmental characteristics will be the opportunity to connect with existing models and standards such as CityGML (Gröger et al 2019) or BIM / IFC (https://www.buildingsmart.org/standards/bsi-

standards/industry-foundation-classes/) that focus on aboveground features, or GeoSciML (OGC 2017) / GroundwaterML (Brodaric 2017) that cover a broad range of geologic and hydrologic phenomena. This will enable standardized, interoperable data to model potentially the entire interconnected built and natural municipal environment from top to bottom at each scale from small local jurisdictions to regional and national extents.

\section{MODEL SCOPE}

Data and models of the built environment are increasingly valuable tools in enabling urban systems to serve their inhabitants and adapt to the significant changes in society, climate, and technology which seem to be coming at us every day. It is visually evident that models of the built environment at or above the ground surface need to be 3 dimensional to best fill that role, but it is often less evident that the substantial proportion of the environment hidden 
from view beneath the surface also has critical 3-dimensional characteristics. We may think of underground utilities as laid out in neat $2 \mathrm{D}$ grids, for example, when we think of them at all. The complicated reality of the underground environment is that utility assets, surface structures supports, underground structures themselves, and all sorts of abandoned material twine around each other at a range of subsurface depths. They in turn are surrounded and supported by intricate units of soil, sediment, debris, bedrock, and groundwater that derive their nature and form from chaotic mixtures of natural and man-made processes. All of this, in contrast to the aboveground environment, can only be viewed directly in excavations, sampled intermittently with boreholes and geophysical methods, or inferred from surface connections. The scarcity and cost of underground data in turn create concerns of security and propriety completely at odds with those of surface assets, such as fire hydrant, that anyone can just walk up to or even view from space.

There are many compelling use cases for high quality data for the underground environment collected at multiple levels of detail, from awareness of accurate utility asset locations and identities for safe excavations, through cost-effective design and construction activities in complexly built up urban areas, to planning for vulnerability and resilience of infrastructure in the face of natural and man-made disasters. There is little question that the value of avoiding damage, delay, injury and cost with good underground data far outstrips the cost and difficult of obtaining it. It is also true that data on the location and disposition of buried utility assets as well as other elements of underground infrastructure, are difficult to collect and maintain, particularly for already buried and rarely maintained assets. Still, present data are most commonly of poor quality or even non-existent, and almost always limited to $2 \mathrm{D}$ point and centerline representations. Much actual knowledge of these assets remains outside of information systems in the heads of the utility workers who last installed or serviced them, inaccessible for other purposes, and vulnerable to departures and retirements. This seems to be as much a cultural as a technical problem, with each asset owner focusing on their own assets and on discovering asset positions as and when needed. While minimizing immediate costs, this can avoid a truth that would be obvious in any 3D visualization: assets whether they be from different eras, owners, or systems are all mixed up together in the subsurface, equally vulnerable to degradation and to natural or man-made disturbances. Integrated datasets and tools for collective awareness should enable each worker to do a better, safer job, although only in the case where employers are able to take a broader view of the value of that awareness.

Besides collection challenges, effective management and utilization of underground data also depend on models and schemas to organize them. Sharing and exchange of such data require standard models that are agreed between data providers and consumers. There are a number of models and standards in present use that are applicable to underground data; they typically differ from each other depending on region of implementation, type of underground asset, target use case, available technology platforms, and legal / policy environment. While unquestionably useful, these often reflect a specific perspective, focus, and priorities that make it difficult for any one of them to provide a holistic awareness of all the natural, human-altered, and man-made elements at each relevant level of detail that in reality compose the underground built environment. The Open Geospatial Consortium (OGC) along with its members and partners have been engaged for several years now (Lieberman \& Ryan, 2017), (Lieberman ed., 2019) in understanding the concepts and issues surrounding underground data in order to provide guidance and standards that might help to address the data and model shortcomings which plague this domain.

\section{MODEL USE CASES}

The following 6 use cases are described in (Lieberman, ed., 2019) to represent distinct requirements for detail, resolution, coverage, model complexity, and capacity to represent change over time, whether observed or predicted.

\subsubsection{Routine street excavations / safe digging (EX)}

In many developed areas, between 30 and 40 excavations are carried out annually per mile of roadway. Urban and even suburban underground spaces are usually a tangle of many different utility lines. For safe digging, most jurisdictions require that utilities share what they know about their assets at the location of any proposed excavation in order to avoid utility strikes, which can cause extensive damage as well as significant costs and delays. In some cases, mark-up crews locate records of questionable currency for the street in question and bring them out into the field in their vehicles. Information sharing consists of each crew making "graffitistyle" sketches on the street with spray paint or chalk. Getting all the utilities to respond often takes days to weeks - if essential records can be located at all. The effectiveness of this process depends upon the often questionable, rarely documented, and even more rarely verified completeness and accuracy of the retrieved records.

If utilities records were in digital formats based on open standards, excavation requests could be addressed from digital submissions by each utility. With the utility data in a common format, The underground situation could be seamlessly integrated by excavators and used to guide their underground planning and tasking. Modern data exchange methods such as wireless communications and mobile devices could then make it possible to assemble utility information on demand, directly in the field, lowering strike risks and speeding the time required to determine safe digging approaches from days or weeks to minutes.

\subsubsection{Planning, design and construction of large-scale building projects (AE)}

Cities and other large urban entities are constantly changing and being reconstructed. There could be a dozen or more new building or rebuilding projects on the drawing boards and in progress at any one time. Clearly there is a vested interest that any of this development activity be as economical as possible, with costs minimized and projects are completed on schedule and in budget. Project planners, engineers and architects can only accomplish this with access to the best possible information on existing, "as built" conditions from which to create their plans and designs. They need to know if existing utilities and characteristics of the underground environment can support the scale and nature of the projects envisioned. In particular, the precise location and dimensions of existing utility assets are needed in order to economically and safely plan building foundations and new building service connections. Such information need to be not only complete and high quality, but also in a form that is straightforward to integrate and analyze. 
Comprehensive and compatible information about existing infrastructure is just the first step for efficient planning and design for major new development. Information about existing and future natural conditions is also key in planning for both development impacts to the environment and built environment vulnerabilities to threats such as flooding, hurricanes, and earthquakes. Good and cost effective designs must account for these contingencies. When data are incomplete, incompatible, and inaccessible, unduly large contract contingencies are needed to cover the uncertainties and significant delays in construction progress typically result. Once construction has started, discoveries of serious and previously unknown conditions in the underground environment all too often require very expensive change orders and long delays. Major projects covering significant areas and extending over multiple years especially need continued access to good, current underground data. Lack of continued access adds yet more additional cost and risk to the project.

\subsubsection{Disaster planning and response (DP)}

Major disaster events (still) do not happen very often, but they eventually do happen, and the failure to anticipate their effects on major urban infrastructure or other components of the built environment end up adding billions in costs as well as exacerbating the resulting impact in displacements, injuries and death to urban populations. Power blackouts, floods, tsunamis and storm surges; earthquakes, tornados, hurricanes and other high wind events; high heat events, fuel explosions, and terrorist attacks particularly cause or are associated with infrastructure failures. Disaster events can lead and have led to the failure of major utility generation, storage, control or transmission facilities serving significant urban areas. Power failures due to storm, flood or heat events may black out entire regions and shut down other critical facilities. Storm surges can flood transit and vehicular tunnels, short-circuit substations and knock out vulnerable below-grade utility assets of all types. Interdependencies between infrastructure networks are particularly problematic; the failure of one system can lead to the cascading failures of others. It is true that not all the damage caused by a disaster event can be anticipated or prevented, but any actual ability to do so depends completely on the rapid availability of high-quality, interoperable, and geospatially enabled data for analyzing disaster vulnerabilities.

High-quality underground utility data that can be integrated to represent large-scale and/or critical transmission, generation, or storage features along with their physical and functional interconnections and dependencies are particularly important. They enable the visualization, analysis, and what-if simulations of the effects of a disaster event or protective strategy, as well as enable rapid reactions to outages and damage. Utility data are also important for identifying single points of failure, interdependencies, and cascading effects. Above-surface feature data along with characteristics of underground environment such as soil permeability and saturation are also needed to examine the effects of disaster scenarios. Major utility features are only a small part of the overall building-by-building, street-by-street utility infrastructure, and concerns for their security are often cited as grounds to oppose data standardization and sharing. The inability to appropriately share, integrate and analyze data for both strategic and street-level infrastructure components, however, typically leaves jurisdictions less rather than more secure, unnecessarily vulnerable and reactive to disaster events rather than properly prepared.

\subsubsection{Utility related emergency response (ER)}

Rapid and accurate assembly of utility information is a significant value even for routine excavations, but is a far more urgent matter when dealing with an emergency. Responders need to know about all the utility assets they may encounter, their capacities and dependencies, before excavation; they also need to know about the locations of utility control features that can rapidly shut off service to critical network segments and service areas in the event of a significant break. They also need to know the consequences of activating controls for other utilities as well as their own. For example, when a water main leak is strongly suspected, a series of control valves must be located and shut off in sequence to stop the flow since a water supply system is a looped rather than a radial network. If some of those valves are electrically actuated, interrupting the power may prevent interrupting the water flow. Data on the properties of on-scene soils and sediments may also help to determine whether flow scouring from a water release could undermine other utility lines and/or nearby building foundations. Slow access to this sort of integrated information, even if it does exist, can lead to more dangerous and costly situations. Stories abound of utility workers at the scene of an incident huddling over paper plans on the hoods of multiple trucks, trying to figure out what might be happening, while it continues to happen all around them.

The value of access to complete, accurate and interoperable underground data via wireless field communications is hard to overstate. It can empower emergency field workers to rapidly understand the nature of a utility problem and to take informed, effective action. Control valves can be quickly located and properly actuated. Excavation of a damaged pipe or conduit can be commenced immediately with confidence that all other utility asset locations in the vicinity are known and can be properly worked around without significant risk of additional damage.

\subsubsection{Private and public utility operations, maintenance, repair and replacement programs (OM)} All utility assets degrade over time, so all functioning utilities have maintenance programs to ensure that their networks continue to function in accordance with regulations and with a minimum of complaints and outages. Many such maintenance operations involve the replacement of old or obsolete infrastructure assets with new, safer and/or higher-capacity components. It is increasingly important for making economically responsible decisions to be able to analyze the performance of individual utility assets, assemblages, and entire networks. This necessarily involves complete and accurate utility feature information including age, material, capacity and location. While installing new gas lines or electric conduit may be expensive, analysis can show that timely upgrades are less expensive than dealing with major service outages when utility components fail or no longer meet demand. Sharing of asset and maintenance activity information can also lead to efficiencies and savings through sharing of street cut and excavation tasks between utilities

Utility responses to reported network issues also depend upon data that relates reported problems to specific utility asset locations and characteristics. Underground environment data, such as earth materials and structures, moisture, effects of other nearby utility components, can also help utility analysts understand where segments of their networks are at greatest risk from threats such as corrosion, material fatigue, and breakage. Active sensors are increasingly in use to monitor 
utility assets in the field and can provide even more layers of information and intelligence to support maintenance and operations decisions.

\subsubsection{Smart Cities, Future Cities (SC)}

New generations of sensors and smart controls attached to underground infrastructure components are transforming monitoring and operation of infrastructure networks as well as revolutionizing the way infrastructure product delivery is managed. In turn, the power and telecommunications infrastructure that these sensors and controls require, will increase utility interdependencies. Innovative smart city technologies are also leading to new types of utility and infrastructure services such as curb-side charging stations for electric vehicles and infrastructure for autonomous vehicle navigation. Safe and cost-effective build-up of underground networks to accommodate these new developments, will require increasingly comprehensive knowledge of infrastructure locations, characteristics, and functionality. Jurisdictions will need to plan for major overhauls of their underground infrastructure and environment to provide new services and respond to new challenges such as climate resilience. An important step in preparing for these changes will be to fully document the infrastructure that is currently in place, and to evolve standard data models for the new services and devices that are on the way. Ignorance of what is already underground will be a substantial barrier to adopting future city innovations.

\section{MODEL DESIGN CRITERIA AND PATTERNS}

The Open Geospatial Consortium along with its members and partners have been engaged for several years now in understanding the concepts and issues surrounding underground data in order to provide guidance and standards that might help to address the data shortcomings which plague this domain. One design criterion of this work has been to draw from the usefulness of existing standards inside and outside of OGC that do a good job with specific aspects of the underground environment for specific purposes, by developing what might be termed an integration or mediation model. This is a standards development approach where common model elements are defined based on mapping to and from elements in existing standards. This enables datasets conforming to those standards to be integrated together to provide a richer picture than that provided by any individual dataset or model.

An important model design technique for supporting broad data interoperability involves creating corresponding model specifications at multiple levels of realization detail. A conceptual model represents the broadest but most general and least detailed level. This level is specified in a conceptual language such as UML that many different implementations and technology platforms are able to conform to. A more detailed, logical level of specification makes use of more specific languages such as SQL that are each able to represent most implementation details across several platforms. Physical models represent the most detailed realization of a standard model, but are typically limited to one technology platform or even one implementation. This approach provides for the greatest feasible commonality and therefore interoperability between implementations at each level of realization detail. It is especially valuable in such a diverse ecosystem as that supporting the wide ranging use cases for underground data.
Another design criterion has been to support representations of the underground environment that match in complexity to specific use cases by taking a modular approach to model detail and capabilities. A conceptual core covers basic geometric representations of underground assets, and a number of extension modules add more specialized assets, more complete geometric and functional representations, and more capabilities in the form of interfaces with other models and perspectives. The modular core and extensions structure makes use of several extension mechanisms, including inheritance, where more specialized elements with specific properties are derived from more general elements with fewer, more general properties. Another mechanism consists of interfaces - sets of properties and/or operations which can be used to add additional capabilities, such as specific geometry types, to wherever in the implemented model schema they may be needed. A third mechanism is linking of additional detail modules through common asset identifier attributes, which is particularly useful for distributed storage and management of specialized or large-scale underground data such as detailed point cloud or voxel representations.

A modular approach to $3 \mathrm{D}$ representation and visualization has been adopted as well. The model core follows the 3D measurement approach being taken in the American Society of Civil Engineers (ASCE) draft "As-Built" specification for recording data on underground structures and assets. This approach focuses on collection of dimensional attributes which are not themselves complete 3D geometries, but which comprise sufficient information (length, height, width, profile, structure type, material, etc.) for a visualization system to reconstruct at least generally an accurate $3 \mathrm{D}$ representation of the structure or asset. This reconstruction can then be used for more realistic visualization but also for quantitative analysis, such as designing new construction to work around the underground volumes occupied by existing structures. An advantage of the measurement approach is that $3 \mathrm{D}$ reconstructions can be more or less schematic depending on the quality and completeness of available data and still retain the important perspective that the underground region is inherently a 3D volume with assets and other features occupying specific depth intervals as well as locations both relative to each other and with respect to vertical earth-fixed datums. Additional modules can support direct 3D representations of the same assets, such as CityGML models, 3D point clouds, or 3D Tiles renderings when such representations are available or have been constructed and the capability exists to maintain them persistently. These explicit representations are becoming increasingly available due to advances in as-built design quality, advances in sensing technologies such as ground penetrating radar, and greater opportunities to survey existing infrastructure during excavations with both imaging and lidar scanning instruments. The measurement approach, however, is likely to remain for the foreseeable future the most widely applicable means of moving to full $3 \mathrm{D}$ awareness of the characteristics and vulnerabilities of the subsurface built environment.

Data terminology is a particular challenge in designing an integration model that may be drawing from many disparate data sources in different organizations, domains, and communities. Even once transformed into a common structure, widely varying terminology can make it difficult to have a common understanding of what is being represented in the model. For this reason, the approach has been taken of incorporating a wide variety of standard codelists for values of model attributes. While presenting in turn a management 
challenge, such codelists provide some guarantee that queries, for example, will return all appropriate data records and not founder on slightly differing spelling or other idiosyncracies.

\section{CONCEPTUAL MODEL ELEMENTS}

The Model for Underground Data Definition and Integration (MUDDI), a comprehensive integration model for underground information that has resulted from this work, is based on mappings from a number of existing standard models for utility and underground information. These include the IMKL model standard (https://www.agiv.be/producten/klip/meer-over/technischedocumentatie/technische-documentatie-imkl) in use in Flanders, which in turn is based on the INSPIRE Utility Networks Standard (http://inspire.ec.europa.eu/theme/us). Other inspiration has included the Singapore Land Authority model (Yan et al 2019). Aspects of utility network connectivity are drawn from the CityGML Utility Network Application Domain Extension (ADE) ( http://en.wiki.utilitynetworks.sig3d.org) which in turn extends the CityGML standard (Gröger et al 2019). As described above, many core entity attributes are also drawn from the draft ASCE "As-built" specification (ASCE 2018), while data quality levels follow those of the existing ASCE 38-02 and PAS 128 standards.

The model packages so far defined in the MUDDI conceptual model are shown in Figure 1. These comprise the MUDDI_Core package, MUDDI_Environment package, MUDDI_Network package, and a package used for adding specific 2D geometry types at the logical level, the MUDDI_Logic2D package.
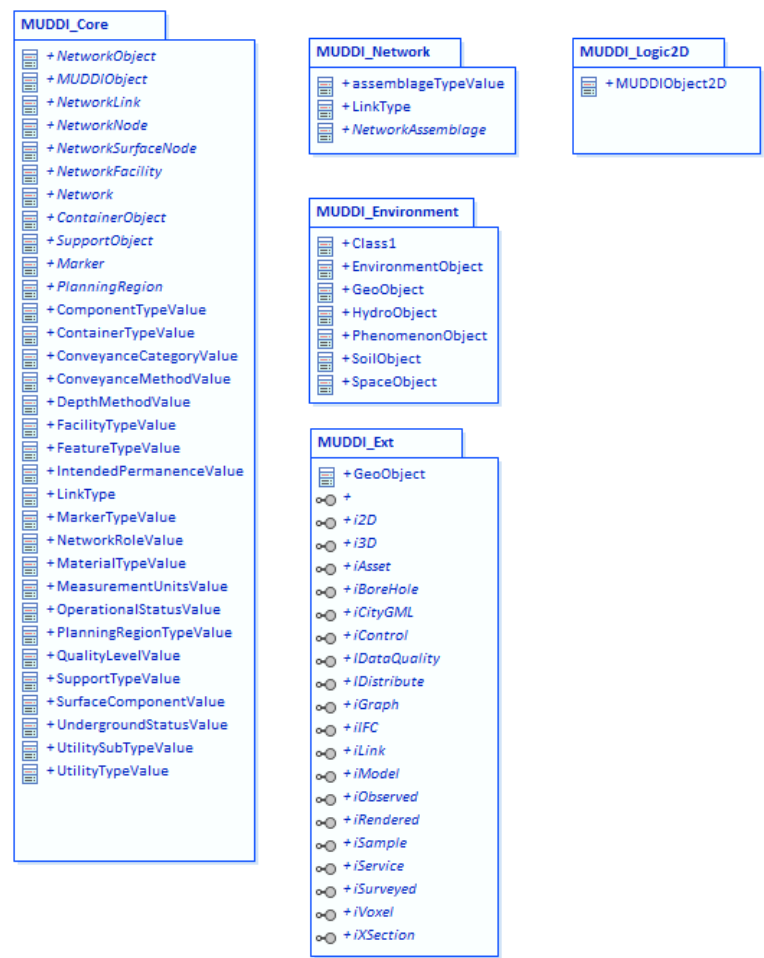

Figure 1. MUDDI UML specification packages

The MUDDI_Core package actually comprises a small number of asset classes, along with an extensive set of codelist definition classes to provide control of terminology used to describe underground assets. Figure 2 shows the core asset classes. A root MUDDIObject class is specialized into classes covering common underground assets such network links and nodes, containers, supports, facilities, and planning regions. A network surface node class provides a basic level of interface between assets which are buried and those installed on or above the surface.

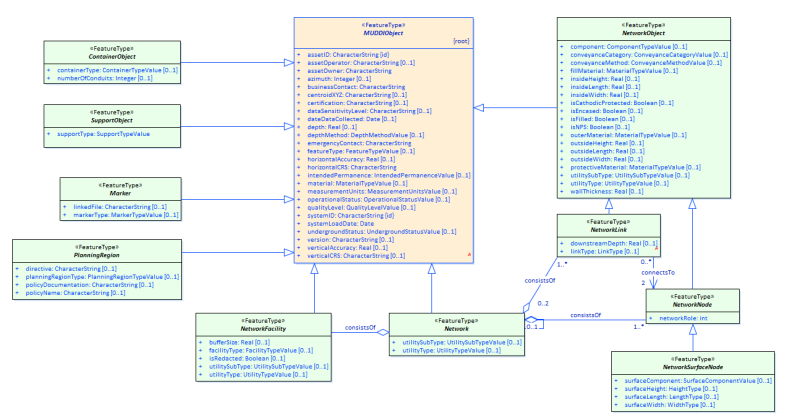

Figure 2. MUDDI Core asset classes

The basic structure of the MUDDI_Environment package is shown in Figure 3, where MUDDIObject is specialized first into an EnvironmentObject and then into separate media such as soil and water.

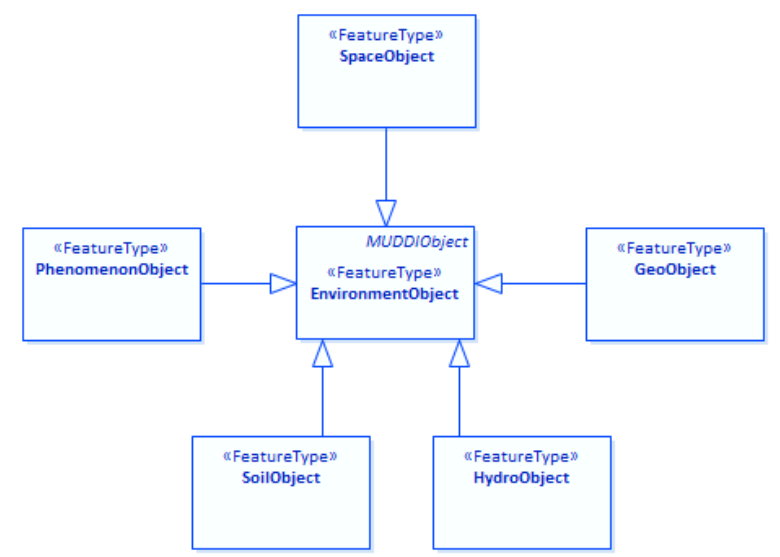

Figure 3. MUDDI_Environment classes

Models such as the CityGML Utility Network ADE, CIMS, and MultiSpeak provide very detailed and/or utility-specific representations of network connectivity that are powerful but complex for simpler applications. The base MUDDI_Network classes shown in Figure 4 provide just a limited profile of such connectivity in order to support initial requirements of some of the more advanced use cases, such as utility asset operational dependencies for disaster vulnerability assessment. 


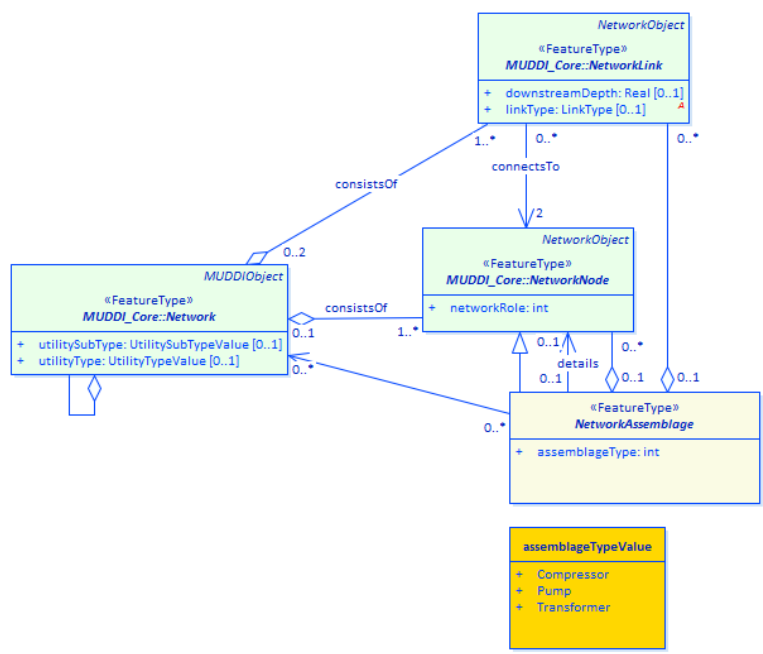

Figure 4. MUDDI_Network classes and link relations

As noted above, the MUDDI conceptual model is intended to provide the broadest level of interoperability between derived logical and physical model realizations that have been and are to be developed to govern model implementations.

\section{IMPLEMENTATION CONSIDERATIONS}

Several prototyping efforts have generated implementations of the MUDDI conceptual model, in particular the NUAR and LUAR pilot projects sponsored by the UK Geospatial Commission and carried out by Ordnance Survey UK and the UK Greater London Authority. These efforts have involved creation of a pilot-specific logical model conforming to the MUDDI conceptual core classes, adding specialized utilitytype classes as well as implementation-specific geometry attributes from the MUDDI logic2D package. Both a physical geodatabase generation script and a GML schema were then generated automatically from the logical model using the ShapeChange utility. This fulfilled two important functions for the NUAR utility asset search and visualization platform: a means of storing utility assets from different providers in a common format, and a file exchange format in which utility data contributions could be provided, optionally by way of a Web API such as OGC API - Features (Portele, et al 2019) . The codelist approach of the MUDDI model provided not only consistency in searching for utility assets by area of interest, but also facilitated the symbolization of asset data on working maps.

As pilots, these and other initiatives have still to address further issues of scale and diversity, for example how to manage model integrity and still maintain codelists across dozens or hundreds of different data providers. Future pilot and prototype activities are likely to address these and other implementation issues, such as incorporation of geotechnical and hydrotechnical environment data into MUDDI datastores.

\section{STANDARDIZATION PROCESS}

An OGC Standards Working Group has now been formed that is set to build on the draft MUDDI model as well as the experiences gained in the NUAR and LUAR pilot projects, in order to develop and standardize a full specification of the model at the conceptual, logical, and physical levels. Another working group objective will be to create a roadmap of critical extension modules, particularly those which support upcoming 3D-4D digital twin technologies for visualization, operation, and simulation. Other advanced use cases for these extensions, such as mixed reality visualization and navigation, are expected to become common as both the demands on our built environment and the data available to manage it continue to expand.

The MUDDI standard specification deliverables to be developed include:

- A conceptual model describing the scope, critical concepts and main relationships that define the model.

- A roadmap specification describing how extensions, interfaces, and specializations to MUDDI should be developed and provide an initial listing of expected modules.

- At least one logical model specification that is consistent with the conceptual model and provides the basis for (potentially automated) generation of one or more implementation specifications.

- At least one implementation specification supporting a specific data language and encoding, such as GML, CityGML, SF-SQL, or GeoJSON.

- (Optional) Mapping and/or extension specifications that describe how to carry out partial or complete data transformations between MUDDI and other relevant models that are proposed or in use for underground data such as CityGML UN ADE, IFC, LandInfra, PipelineML, CIM, Multispeak, etc.

As an open standard, MUDDI will be available both to be extended for additional use cases and profiled for more specific underground environment domains. Conformance to the base models will need, however, to be demonstrated in order to preserve the harmonization and interoperability benefits that a common integration model can provide.

\section{CONCLUSIONS}

A series of collaborative investigation, benefit analysis, and design activities has led to development of a draft conceptual integration model for elements of the underground built environment. The MUDDI model incorporates a number of design principles and patterns, in particular a modular coreextension structure, in order to best balance fitness for particular use cases with flexibility to coordinate with existing standard data models and future technologies for underground data collection and utilization. Several pilot implementations have demonstrated the feasibility of the MUDDI model for integrating utility asset data from many different providers to support safe digging operations. A 3-level model specification approach provided distinct data interoperability benefits.

Based on the initial success and promise of the MUDDI model, an OGC Standards Working Group is being formed to further develop and specify the model in order to publish it as an open implementation standard, making it widely available for implementation and adoption that support efforts to improve knowledge of the urban underground environment.

\section{ACKNOWLEDGEMENTS}

The authors would like to thank Ordnance Survey GB (https://www.ordnancesurvey.co.uk) and 1Spatial (https://1spatial.com/) for sponsoring the publication of this paper. 


\section{REFERENCES}

J. Lieberman ed., 2019. MUDDI Model for Underground Data Definition and Integration Engineering Report, http://docs.opengeospatial.org/per/17-090r1.html

C. Portele, P.Vretanos, C. Heazel, 2019.

OGC API - Features - Part 1: Core https://www.opengeospatial.org/standards/ogcapi-features

G. Gröger, T. Kolbe, C. Nagel, K.Häfele, 2019. OGC City Geography Markup Language (CityGML) Encoding Standard, https://www.ogc.org/standards/citygml

OGC, 2017. OGC Geoscience Markup Language 4.1 (GeoSciML) https://docs.opengeospatial.org/is/16-008/16008.html

J. Lieberman and A. Ryan, ed's, 2017. OGC Underground Infrastructure Concept Study Engineering Report, http://docs.opengeospatial.org/per/17-048.html

B. Brodaric, 2017. OGC WaterML 2: Part 4 GroundWaterML 2 (GWML2), http://docs.opengeospatial.org/is/16-032r2/16-032r2.html

American Society of Civil Engineers, 2018. White Paper on ASCE "Standard Guideline for Recording and Exchanging Utility Infrastructure Data", https://www.asce.org/uploadedFiles/Technical_Areas/Constr uction_Engeering/Content_Pieces/as-built-standards-

whitepaper.pdf

Yan, Jingya \& Jaw, Siow Wei \& Soon, Kean \& Wieser, Andreas \& Schrotter, Gerhard, 2019. Towards an Underground Utilities 3D Data Model for Land Administration. Remote Sensing. 11. 1957. 10.3390/rs11171957. 